\title{
Utilidad de la ecografía y del eco- doppler color en pacientes de alto riesgo obstétrico
}

\author{
Usefulness of ultrasound and color Doppler ultrasound in high-risk \\ obstetric patients \\ Utilidade do ultrassom e do ultrassom Doppler colorido em pacientes \\ obstétricas de alto risco
}

Marcelo De Agostini
ORCID: 0000-0002-5285-6594 Ginecólogo. Profesor Agregado Clínica Ginecotocológica B. Jefe Unidad Ecografía. Hospital de Clínicas. UdelaR.

Rosario Morán ORCID: 0000-0002-4171-8460 Ginecóloga. Ex Asistente Clínica Ginecotocológica B. Hospital de Clínicas. UdelaR.

Cristina Cordano ORCID: 0000-0002-9453-0613 Ginecóloga. Profesora Agregada Clínica Ginecotocológica B. Unidad de Ecografía. Hospital de Clínicas.

UdelaR

Florencia Garat ORCID: 0000-0003-4232-9704 Ginecóloga. Profesora Adjunta Clínica Ginecotocológica B. Unidad de Ecografía. Hospital de Clínicas.

UdelaR.

Resumen: La ecografía obstétrica, nos permite determinar con adecuada precisión la biometría fetal y realizar el seguimiento de su curva de crecimiento en función de la edad gestacional. El Eco-Doppler Feto-Placentario, permite el estudio del flujo sanguíneo materno-fetal y de la circulación placentaria de forma no invasiva, inocua y reproducible. Su eficacia ha sido demostrada en el control del embarazo de alto riesgo obstétrico, logrando una reducción de la mortalidad perinatal del $49 \%$. El mismo evalúa la circulación útero - placentaria (arterias uterinas y arteria umbilical), la circulación fetal arterial (arteria cerebral media) y la venosa (Ductus Venoso, Vena Cava Inferior, Vena Umbilical). Identificar el grupo de pacientes con trombofilia, que requieren un control ecográfico más estricto es fundamental para lograr el beneficio con el tratamiento médico.

Palabras clave: ecografía obstétrica, eco-doppler, síndrome antifosfolípidos.

Abstract: Obstetric ultrasound allows us to determine with adequate precision the fetal biometry and to monitor its growth curve based on gestational age. The Feto-Placental Eco-Doppler allows the study of maternal-fetal blood flow and placental circulation in a non-invasive, safe and reproducible way. Its efficacy has been demonstrated in the control of high-risk obstetric pregnancy, achieving a $49 \%$ reduction in perinatal mortality. It evaluates the uterine-placental circulation (uterine arteries and umbilical artery), the fetal arterial circulation (middle cerebral artery) and the venous circulation (Ductus Venoso, Inferior Vena Cava, Umbilical Vein). Identifying the group of patients with thrombophilia, who require stricter ultrasound control is essential to achieve benefit with medical treatment.

Key words: obstetric ultrasound, ultrasound Doppler, antiphospholipid syndrome.

Resumo: A ultrassonografia obstétrica permite determinar com precisão adequada a biometria fetal e monitorar sua curva de crescimento com base na idade gestacional. O Eco-Doppler Feto-Placental permite o estudo do fluxo sanguíneo materno-fetal e da circulação placentária de forma não invasiva, segura e reprodutível. Sua eficácia foi demonstrada no controle da gravidez obstétrica de alto risco, alcançando uma redução de $49 \%$ na mortalidade perinatal. Avalia a circulação útero-placentária (artérias uterinas e artéria umbilical), a circulação arterial fetal (artéria cerebral média) e a circulação venosa (Canal Venoso, Veia Cava Inferior, Veia Umbilical). Identificar o grupo de pacientes com trombofilia, que necessita de controle ultrassonográfico mais rígido, é essencial para obter benefício com o tratamento médico.

Palavras-chave: ultrassom obstétrico, ultrassom Doppler, síndrome antifosfolipídeo.

Recibido: 06/02/2021 - Aceptado: 31/05/2021

Hospital de Clínicas. Facultad de Medicina. UdelaR.

Correspondencia: E-mail: maristevenazzi@gmail.com 
Uno de los principales objetivos del control prenatal es proporcionar información acerca del bienestar del binomio materno-fetal, para lo cual entre otros parámetros es muy importante el estudio del crecimiento fetal y su hemodinamia.

Tal como se ha discutido en anteriores revisiones, la trombofilia hereditaria tiene escasa o ausente asociación con la morbilidad obstétrica por lo que cuando mecionamos "trombofilias adquiridas" nos estamos refiriendo al Síndrome Antifosfolipídico (SAF) excepto que se aclare específicamente.

\section{Ecografía Obstétrica}

La ecografía obstétrica, utilizada como instrumento de control, es inocua, no invasiva, de bajo costo y reproducible. La misma nos permite determinar con adecuada precisión la biometría fetal y realizar el seguimiento de su curva de crecimiento en función de la edad gestacional, con la posibilidad de elaborar "curvas"1-4.

En base a estas curvas, es posible la detección precoz de la restricción del crecimiento fetal (RCF), que comprende aquellos fetos que no pueden alcanzar su potencial de crecimiento predeterminado y se asocian a mayor morbi-mortalidad perinatal como ya ha sido ampliamente demostrado ${ }^{5}$. De los parámetros biométricos fetales que se pueden evaluar mediante ecografía, el "perímetro abdominal" ha demostrado ser el de mayor sensibilidad y especificidad para la detección de la $\mathrm{RCF}^{3,6,7}$.

El estudio ecográfico obstétrico debe incluir además de la biometría fetal, la valoración del líquido amniótico (cantidad) y de la placenta (grado de maduración, decolamientos, hematomas); elementos éstos que también pueden mostrar cambios cuando se altera el crecimiento y la salud fetal.

\section{Eco Doppler Feto-Placentario}

El Eco-Doppler Feto-Placentario, permite el estudio del flujo sanguíneo materno-fetal y de la circulación placentaria de forma no invasiva, inocua y reproducible. Su eficacia ha sido demostrada en el control del embarazo de alto riesgo obstétrico, logrando una reducción de la mortalidad perinatal del $49 \%$ vinculada a la hipoxia crónica, así como otras complicaciones y costos vinculados al embarazo y parto ${ }^{8-11}$.

El Eco-Doppler Feto-Placentario evalúa la circulación útero - placentaria (arterias uterinas y arteria umbilical), la circulación fetal arterial (arteria cerebral media ACM) y la venosa (Ductus Venoso, Vena Cava Inferior, Vena Umbilical) ${ }^{11,12}$.

\section{a) Arterias Uterinas}

Las arterias uterinas sufren cambios propios del embarazo, a lo largo del mismo, que llevan a una disminución gradual de su resistencia hasta las 24 - 26 semanas, logrando en condiciones normales un flujo de baja resistencia que se mantiene hasta el final del embarazo.

Son cambios patológicos los aumentos de la resistencia en estos vasos o la persistencia de Notch (incisura protodiastólica) del lado placentario o bilateral ${ }^{13}$. Si bien la descripción original del Notch se define como la desaceleración de la onda de al menos $50 \mathrm{~Hz}$ por debajo de la velocidad sistólica máxima, en la práctica, éstos se valoran subjetivamente (se ven o no se ven) y por tanto se interpreta como un signo cualitativo de la onda ${ }^{3}$. Cuando existe Notch, la resistencia vascular a ese nivel está aumentada. Distintos autores han buscado su cuantificación.

Actualmente, en la valoración de las arterias uterinas se recomienda utilizar el índice de Pulsatilidad promedio entre ambas arterias, tomando como punto de corte el percentil 95 para la edad gestacional ${ }^{1,2}$.

La persistencia de Notch en la segunda mitad del embarazo o el aumento de la resistencia en los vasos uterinos (mayor del percentil 95) es un elemento de alerta y por tanto de vigilancia de la salud fetal ${ }^{14}$.

Siguiendo las recomendaciones internacionales, se plantea el uso del promedio del índice de Pulsatilidad en las arterias uterinas, considerando más su elevación que la presencia o ausencia de Notch ${ }^{4}$. De esta manera, se le debe otorgar mayor importancia para la toma de decisiones, al aumento del índice de Pulsatilidad en la curva o a su valor por encima del percentil 95, que al Notch ${ }^{15}$. 


\section{b) Arteria Umbilical}

El índice de Pulsatilidad es el parámetro que valora mejor el aumento en la resistencia vascular placentaria, lo cual ha sido demostrado en múltiples estudios del síndrome Pre eclampsiaeclampsia. Éste debe tomarse en cuenta cuando se realiza el Doppler de la Arteria Umbilical.

No hay evidencia de buena calidad sobre el papel del ecodoppler en pacientes con trombofilias exclusivamente.

La arteria umbilical evalúa la resistencia placentaria. La insuficiencia placentaria se refleja en la disminución de los flujos de fin de diástole en éste vaso, lo que corresponde a un aumento de la resistencia a nivel placentario; esto constituye un signo de hipoxia fetal y plantea la necesidad de vigilancia estricta. La ausencia de flujo de fin de diástole o el flujo reverso son indicadores que se asocian con una mortalidad perinatal elevada ${ }^{12,16,17}$.

En los fetos con restricción del crecimiento de causa placentaria, es posible ver estas alteraciones de la onda de flujo en la arteria umbilical; siendo la elevación del índice de Pulsatilidad en éste vaso, el parámetro para su seguimiento. El feto ante la hipoxia crónica, reacciona poniendo en juego mecanismos compensadores con el fin de preservar órganos nobles. En estudios prospectivos, ha sido demostrado que éste efecto protector alcanza su máxima intensidad 2 a 3 semanas antes de que se presenten alteraciones en el monitoreo fetal o cardiotocograma basal ${ }^{12,16-18}$.

\section{c) Arteria Cerebral Media}

La aparición de vasodilatación en la Arteria Cerebral Media fetal (ACM), se considera un signo de alerta y vigilancia estricta. Cuando desaparece (retorna a valores de alta resistencia), se considera de alarma, dado que se corresponde con fetos graves, con acidosis y riesgo de muerte fetal inminente.

En la toma de decisiones obstétricas, se debe considerar la relación de los índices de Pulsatilidad cerebro/umbilical. Éste índice, ha mostrado ser el mejor predictor de riesgo de morbi-mortalidad perinatal. La relación se altera antes, que se altere alguno de sus componentes por separado.

\section{d) Ductus Venoso, Vena Umbilical y Vena Cava Inferior}

La alteración de los flujos venosos es un signo tardío y marca el agotamiento de los mecanismos compensadores, correspondiendo a acidosis, insuficiencia cardíaca, los que preceden a la muerte fetal.

De las alteraciones venosas valoradas en el Doppler se destacan: flujos ausentes o reversos en Vena Cava Inferior y Ductus Venoso, lo cual representa un aumento del índice de Pulsatilidad; y a nivel de la vena umbilical flujo pulsátil (éste último es un signo premortem).

El hallazgo de un flujo alterado en cualquiera de estos vasos venosos se correlaciona con un $\mathrm{pH}$ menor a 7.2. Las alteraciones en el Ductus Venoso, preceden en días la muerte fetal9,19-21.

\section{Control fetal y alto riesgo obstétrico}

Como hemos expresado anteriormente, no todas las "trombofilias" se asocian a complicaciones materno-fetales y perinatales, por lo cual deben identificarse claramente aquéllas pacientes que requieran un control más estricto y eventualmente se beneficien de un tratamiento médico.

En nuestro medio, las pacientes con SAF y mala historia obstétrica son generalmente consideradas de alto riesgo y por lo tanto son sometidas indefectiblemente a exhaustivo control de salud fetal. Sin embargo, esta conducta está basada en evidencia indirecta, extrapolada de estudios que demostraron asociación entre las pruebas de salud fetal y la morbimortalidad perinatal pero en poblaciones específicas (por ejemplo, Restricción del Crecimiento Fetal, síndrome Pre-Eclampsia-eclampsia) ${ }^{4}$, no precisamente en poblaciones con trombofilia.

Se ha determinado que cuando el SAF se asocia con malos resultados obstétricos, como la presencia de Restricción del Crecimiento Fetal, la utilización de la Ecografía Doppler tiene su máxima efectividad, aumentando el valor predictivo positivo y la posibilidad de detectar poblaciones con alteración de la salud fetal. 
Cuando el Doppler se utiliza para valorar la salud fetal en ausencia de morbilidad obstétrica el valor predictivo positivo desciende drásticamente y se pone en duda la efectividad del estudio Doppler.

La revisión bibliográfica actualizada a la fecha, nos demuestra que aún no existen publicaciones que nos permitan realizar recomendaciones basadas en buena evidencia y con un claro beneficio para las pacientes portadoras de trombofilia hereditaria sin morbilidad obstétrica.

De todas formas, como durante el embarazo aconsejamos realizar screening de riesgo Pre-eclampsia-eclampsia con Doppler de Arterias Uterinas, a toda la población embarazada durante la Ecografía de la semana 11-14 y 20-24, las pacientes que presenten alteraciones de las resistencias en las arterias uterinas, se beneficiarán del tratamiento profiláctico o del estricto control presenten o no algún tipo de trombofilia ${ }^{22}$. En pacientes con SAF u otros factores de riesgo vascular obstétrico sin alteraciones del crecimiento fetal ni alteraciones en las Arterias Uterinas durante las semanas 11-14 y 20-24, se podría comenzar a realizar eco-Doppler a partir de las 26-28 semanas $^{3}$, ya que una alteración a esta edad gestacional tendría implicancia patológica y se podría actuar en consecuencia. A esta edad gestacional, también puede existir un cambio normal hemodinámico, que puede generar errores en la interpretación del estudio y la consiguiente iatrogenia terapéutica ${ }^{3,4}$, por lo que insistimos que el estudio debe realizarlo un técnico debidamente capacitado y eventualmente confirmar los hallazgos en un tiempo prudencial.

En cuanto al manejo del tratamiento con anticoagulantes y el control mediante EcoDoppler Feto-Placentario, no se han publicado estudios de buena calidad que sugieran que el tratamiento con anticoagulantes mejore los resultados clínicos $^{23}$.

A nivel internacional no existen guías clínicas específicas para el seguimiento ecográfico ni con Doppler Feto-Placentario de los embarazos complicados con trombofilia adquirida. Sí se sugiere el estricto control ecográfico del crecimiento fetal.

Las evidencias relacionadas al control de la salud fetal y extrapolada de poblaciones de alto riesgo obstétrico nos permiten clasificarla como evidencia de buena calidad asociada con beneficio (1A y $1 \mathrm{~B})$

\section{Recomendaciones}

El Eco Doppler Feto placentario es útil en la valoración de la salud fetal, principalmente en embarazos de alto riesgo obstétrico.

La persistencia de Notch en el $3^{\circ}$ trimestre o del Índice de resistencias elevado en las arterias uterinas es un elemento de alerta y por tanto de vigilancia de la salud fetal.

Actualmente se otorga mayor importancia para la toma de decisiones al aumento del índice de Pulsatilidad más que a la existencia de Notch.

La aparición de vasodilatación en la Arteria Cerebral Media se considera un signo de alerta y para estricto control fetal. En la toma de decisiones obstétricas, se debe considerar la relación de los índices de Pulsatilidad cerebro / umbilical.

La alteración de los flujos venosos fetales es un elemento tardío .

La solicitud de Eco Doppler Feto Placentario en pacientes con trombofilia aislada, sin repercusión sobre el crecimiento fetal, no ha demostrado mejores resultados perinatales, sin embargo cuando se asocia con la presencia de Restricción del Crecimiento presenta su máxima efectividad en la detección de alteraciones de la salud fetal.

Edad gestacional para seguimiento con Eco Doppler Feto-Placentario: Aconsejamos realizar rutinariamente Eco Doppler de Arterias Uterinas entre las semanas 11-14 y 20-24 para screening de riesgo de Pre-eclampsia - eclampsia, a toda la población embarazada En pacientes con SAF u otros factores de riesgo vascular obstétrico, sin alteraciones del crecimiento fetal ni alteraciones en las Arterias Uterinas durante las semanas 11-14 y 20-24, recomendamos comenzar a realizar eco-Doppler a partir de las 26-28 semanas, ya que una alteración a esta edad gestacional tendría implicancia patológica y se podría actuar en consecuencia.

No recomendamos el uso de la ecografía Doppler para guiar el tratamiento anticoagulante. 


\section{Bibliografía}

1- Campbell S, Dewhurst CJ. Diagnosis of the small-for-dates fetus by serial ultrasonic cephalometry. Lancet. 1971;2(7732):1002-6.

2- Fescina RH, Ucieda FJ, Cordano MC, Nieto F, Tenzer SM, López R. Ultrasonic patterns of intrauterine fetal growth in a Latin American country. Early Hum Dev. 1982 Jul;6(3):239-48. doi: 10.1016/03783782(82)90116-5

3- Royal College of Obstetricians and Gynaecologists. The Investigation and Management of the Smallfor-Gestational-Age Fetus. London: RCOG; 2014. (Green-top Guideline No. 31)

4- Delgado L, Cordano MC, Botto Núñez G, Noria A, D’Avenia J, Martínez Moreno M, et al. Comparacion de patrones ecográficos de crecimiento fetal en embarazadas normales. Rev. Latin. Perinat. 2017;6(3):239-248.

5- Resnik R. Intrauterine growth restriction. Obstet. Gynecol. 2002;99(3):490-496.

6- Cordano MC, Comte E, Bessis R, Papiernik E. Longitudinal follow-up of 100 patients at risk of intrauterine growth retardation: comparison of diagnosis in two periods. J. Perinat. Med. 1988;16(1):616.

7- American College of Obstetricians and Gynecologists' Committee on Practice Bulletins-Obstetrics and the Society forMaternal-FetalMedicin. ACOG Practice Bulletin No. 204: Fetal Growth Restriction. Obstet Gynecol. 2019 Feb;133(2):e97-e109. doi: 10.1097/AOG.0000000000003070

8- Campbell S, Wilkin D. Ultrasonic measurement of fetal abdomen circumference in the estimation of fetal weight. Br J Obstet Gynaecol. 1975 Sep;82(9):689-97. doi: 10.1111/j.1471-0528.1975.tb00708.x.

9- Alfirevic Z, Stampalija T, Dowswell T. Fetal and umbilical Doppler ultrasound in high-risk pregnancies. Cochrane Database Syst. Rev. 2017;2017(6):CD007529.

10- Cnossen JS, Morris RK, ter Riet G, Mol BW, van der Post JA, Coomarasamy A, et al. Use of uterine artery Doppler ultrasonography to predict pre-eclampsia and intrauterine growth restriction: a systematic review and bivariable meta-analysis. CMAJ. 2008 Mar 11;178(6):701-11. doi: 10.1503/ cmaj.070430.

11- Gagnon R, Van den Hof M; Diagnostic Imaging Committee, Executive and Council of the Society of Obstetricians and Gynaecologists of Canada. The use of fetal Doppler in obstetrics. J Obstet Gynaecol Can. 2003 Jul;25(7):601-14; quiz 615-6. English, French.

12- Baschat AA, Gembruch U, Harman CR. The sequence of changes in Doppler and biophysical parameters as severe fetal growth restriction worsens. Ultrasound Obstet. Gynecol. 2001;18(6):571577.

13- Becker R, Vonk R. Doppler sonography of uterine arteries at 20-23 weeks: Depth of notch gives information on probability of adverse pregnancy outcome and degree of fetal growth restriction in a low-risk population. Fetal Diagn. Ther. 2010;27(2):78-86.

14- Harrington K, Cooper D, Lees C, Hecher K, Campbell S. Doppler ultrasound of the uterine arteries: The importance of bilateral notching in the prediction of pre-eclampsia, placental abruption or delivery of a small-for-gestational-age baby. Ultrasound Obstet. Gynecol. 1996;7(3):182-188.

15- Nicolás Sáez O, Jorge Carvajal C. Tamizaje y prevención de preeclampsia guiado por doppler de arterias uterinas: Revisión sistemática de la literatura. Rev. Chil. Obstet. Ginecol. 2012;77(3):235-242.

16- Bilardo CM, Wolf H, Stigter RH, Ville Y, Baez E, Visser GH, et al. Relationship between monitoring parameters and perinatal outcome in severe, early intrauterine growth restriction. Ultrasound Obstet. Gynecol. 2004;23(2):119-125.

17- Bilardo CM, Wolf H, Stigter RH, Ville Y, Baez E, Visser GH, et al. Progression of Doppler abnormalities in intrauterine growth restriction. Ultrasound Obstet. Gynecol. 2008;32(2):160-167.

18- Hecher K, Bilardo CM, Stigter RH, Ville Y, Hackelöer BJ, Kok HJ, et al. Monitoring of fetuses with intrauterine growth restriction: A longitudinal study. Ultrasound Obstet. Gynecol. 2001;18(6):564-570.

19- Morris RK, Selman TJ, Verma M, Robson SC, Kleijnen J, Khan KS. Systematic review and metaanalysis of the test accuracy of ductus venosus Doppler to predict compromise of fetal/neonatal wellbeing in high risk pregnancies with placental insufficiency. Eur J Obstet Gynecol Reprod Biol. 2010 Sep;152(1):3-12. doi: 10.1016/j.ejogrb.2010.04.017.

20- Morris RK, Malin G, Robson SC, Kleijnen J, Zamora J, Khan KS. Fetal umbilical artery Doppler to predict compromise of fetal/neonatal wellbeing in a high-risk population: systematic review and bivariate meta-analysis. Ultrasound Obstet Gynecol. 2011 Feb;37(2):135-42. doi: 10.1002/uog.7767. 
21- Brar HS, Platt LD. Reverse end-diastolic flow velocity on umbilical artery velocimetry in highrisk pregnancies: an ominous finding with adverse pregnancy outcome. Am. J. Obstet. Gynecol. 1988;159(3):559-61.

22- De Agostini M, Alonzo I. Guías para la Aplicación Clínica del Ultrasonido en Ginecología y Obstetricia. Montevideo: Fundación peluffo Giguens, 2017.

23- Magriples U, Ozcan T, Karne A, Copel JA. The effect of anticoagulation on antenatal ultrasound findings in pregnant women with thrombophilia. J. Matern. Neonatal Med. 2006;19(1):27-30.

\section{Aporte de cada autor al trabajo}

Marcelo De Agostini: Concepción y diseño del trabajo, recolección de datos, redacción y revisión crítica del manuscrito.

Rosario Morán: Concepción y diseño del trabajo, recolección de datos, redacción y revisión crítica del manuscrito.

Cristina Cordano: Concepción y diseño del trabajo, recolección de datos, redacción y revisión crítica del manuscrito.

Florencia Garat: Concepción y diseño del trabajo, recolección de datos, redacción y revisión crítica del manuscrito. 